\title{
Recherches récentes sur la cavitation à l'Institut de mécanique de Grenoble
}

\author{
J. M. Michel
}

\section{Introduction}

Les recherches menées à l'Institut de mécanique de Grenoble sur les écoulements cavitants ou ventilés ont débuté en 1967, après qu'eut été construit le tunnel hydrodynamique de l'Université de Grenoble [1]. A cette époque, en France comme dans d'autres pays, la nécessité était apparue de promouvoir les recherches dans le domaine de l'hydrodynamique rapide : le développement des machines hydrauliques d'une part, l'intérêt des Marines nationales pour les navires rapides d'autre part, conduisaient à l'étude des conditions limites des écoulements subcavitants et à l'analyse du comportement des écoulements cavitants ou ventilés. Ce secteur de l'activité de recherche a été pris en charge par la Direction des recherches et moyens d'essais (DRME) puis par la Direction des recherches, études et techniques (DRET) du Ministère de la Défense. La décision de constituer à Grenoble une équipe de recherche universitaire fut prise en 1964, compte tenu en particulier des travaux antérieurs de J. DoDU dans le domaine des jets rapides [2] et de l'expérience de la cavitation acquise déjà au sein de la Sogreah ([3] par exemple). Les programmes de recherches ont été depuis lors inspirés en grande partie par la Marine nationale. Schématiquement, deux thèmes principaux ont été abordés: celui du fonctionnement des ailes ventilées, lié au projet de constructions d'hydroptères rapides $[4,5]$, puis à partir de 1980 environ, l'analyse physique de la cavitation développée sur des aubages d'hélices [6, 7]. Les travaux que nous présentons maintenant sont relatifs à ce dernier thème. Financés principalement par la DRET, ils ont été l'occasion d'une concertation étroite avec les établissements Alsthom-ACB-CERG. Nous les regroupons autour des deux sous-thèmes suivants, auxquels sont associés deux moyens d'essais différents :

- la cavitation développée, étudiée sur le tunnel hydrodynamique en configuration bi-dimensionnelle,

- l'érosion de cavitation, étudiée sur une boucle à mercure.

\section{Cavitation développée}

\subsection{Le tunnel hydrodynamique}

Ce moyen d'essai a été décrit d'abord en [1] et, très récemment, en [8]. Il est muni à sa partie supérieure de deux circuits desservant deux sections d'essais : la section $\mathrm{n}^{\circ} 1$ sera pourvue prochainement d'une veine rectangulaire en charge tandis que la section $n^{\circ} 2$ est équipée d'un canal à surface libre (hauteur $400 \mathrm{~mm}$, largeur $120 \mathrm{~mm}$, longueur $1600 \mathrm{~mm}$ ), dans lequel tous les essais à surface libre présentés ici ont été effectués. Les principales caractéristiques de l'écoulement dans ce canal sont les suivantes: Vitesse $V$ de l'eau comprise entre 2,5 et $13 \mathrm{~m} / \mathrm{s}$; pression minimale $p_{0}$ à la surface libre du canal

\section{Recent researches relating to cavitation carried out at the Grenoble Institute of Mechanics.}

Two themes combining two various test means are covered:

The developed cavitation studied in the IMG's hydrodynamic tunnel and the cavitation erosion studied on a mercury loop.

The developed cavitation study includes : the analysis relating to the separation of a cavity from a wall, the unsteady cavitation per pocket, the interaction between germs, the boundary layer and the cavity (this study is dealt with in a separate report), and the experimental study of partial cavitation.

Erosion tests were carried out in collaboration with the CERG and EDF with a view to clarifying the rules of similarity.

Finally, a special effort has been made so as to develop and calibrate miniaturized pressure sensors specially adapted to measure instantaneous excess pressures due to structural implosion. 
1.

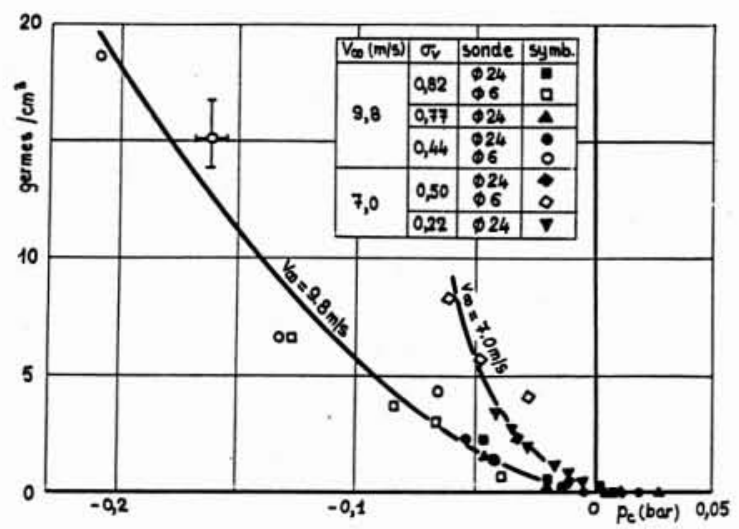

2.

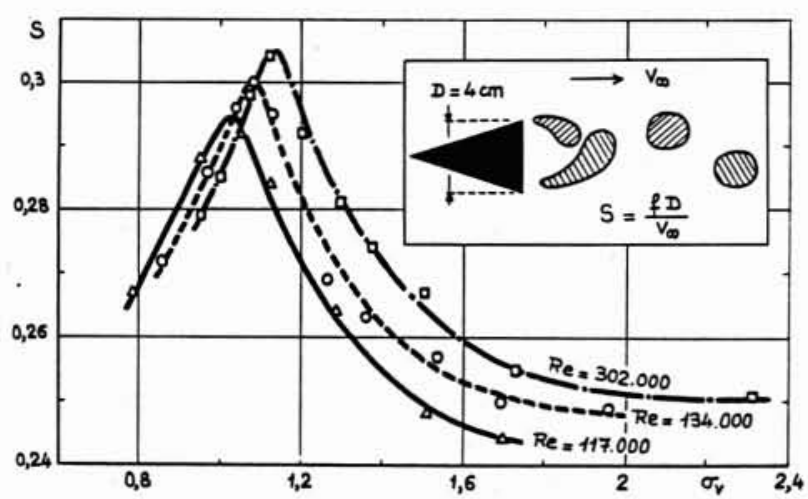

1. Histogramme cumulé de population de germes.

2. Modification du nombre de Strouhal par le développement de la cavitation dans le sillage d'un coin.

3. Carte des régimes cavitants dans le plan $\left(\alpha, \sigma_{v}\right)$. Cas du profil $N A C A$ 16012. $\mathrm{Re}=V c / v=10^{6}$.

4. Début de cavitation sur une aile symétrique (corde $10 \mathrm{~cm})$. Les mêmes tendances sont observées à $\mathrm{Re}=2 \times 10^{6}$ et $2,8 \times 10^{6}$ (Essais EPFL).
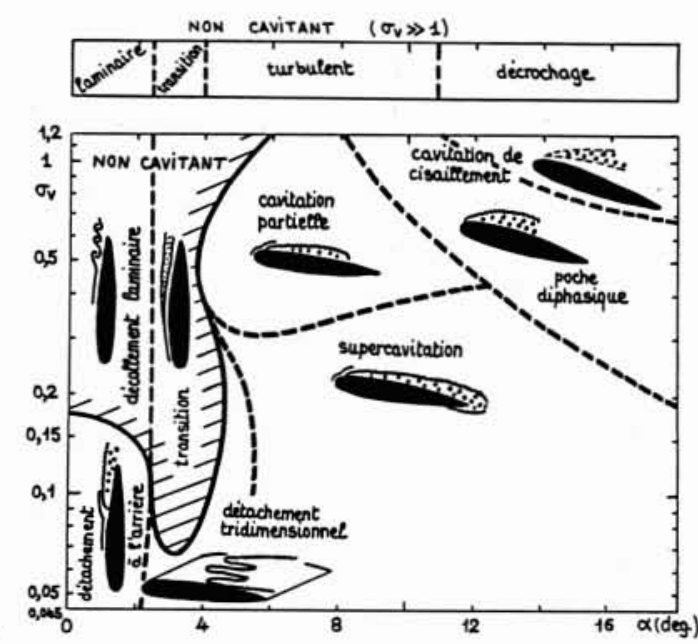

3.

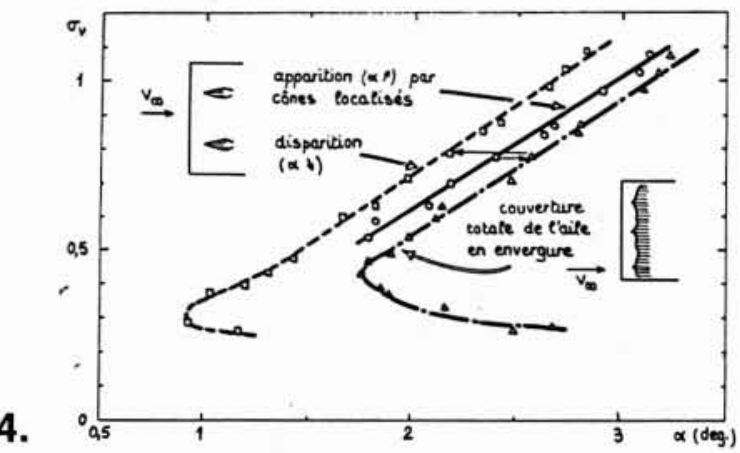

En 1986, le tunnel hydrodynamique a été équipé d'un système complet de contrôle de la concentration en germes de l'eau de l'écoulement, comprenant un dispositif d'injection des germes et un dispositif de détermination des histogrammes des germes injectés en fonction de leur pression critique. Ce système, qui a été réalisé par Alsthom-ACB-CERG, est décrit en [8] et [9]. On note particulièrement les points suivants : la grande dynamique de l'injection et de la mesure des germes (de 0,02 à 20 germes par $\mathrm{cm}^{3}$ ), le caractère représentatif des échantillons prélevés (un point de mesure résulte de l'examen d'une centaine de litres d'eau), la répétabilité des mesures, enfin leur bonne correspondance avec l'examen cinématographique des figures de cavitation par bulles séparées sur le profil placé dans le canal d'essai. Un exemple d'histogramme cumulé, donnant le nombre de germes par $\mathrm{cm}^{3}$, dont la pression d'éclatement est égale ou supérieure à $P_{\text {ccl. }}, n_{g}\left(P_{\text {ecl. }}\right)$, est donné sur la figure 1 . 


\subsection{Cavitation de sillage}

La cavitation développée à l'aval de cylindres de diamètres 1,2 et $5 \mathrm{~cm}$ et à l'aval d'un coin triangulaire (base $4 \mathrm{~cm}$, hauteur $10 \mathrm{~cm}$ ) tenu à incidence moyenne nulle a été étudiée par FrANC [6,10]. La cavitation rend apparentes les principales structures tourbillonnaires d'un sillage dont le cœur est le siège de dépressions plus ou moins importantes: tourbillons alternés de BénardKarman, tourbillon en formation à la base de l'obstacle, tourbillons issus de la couche limite sur le corps, qui se déversent dans le sillage proche, enfin tourbillons secondaires à peu près alignés sur l'écoulement moyen et répartis le long de l'envergure du corps. On doit noter que la cavitation ne joue pas seulement le rôle de visualisant passif de l'écoulement subcavitant préexistant, mais que celui-ci doit composer avec la dynamique propre aux implosions et explosions de poches de vapeur, dont les temps caractéristiques sont très petits. Il en résulte une modification appréciable des caractères globaux de l'écoulement. Par exemple, la figure 2 montre la modification du nombre de Strouhal engendrée par le développement de la cavitation. Un pic est visible au voisinage de la valeur $\sigma_{v}=1,1$ du nombre de cavitation du corps, défini ici, comme dans la suite, par $\sigma_{v}=\sigma_{v_{0}}+2 g h / V^{2}$, où $h$ est la profondeur d'immersion du corps (usuellement, $h=0,20 \mathrm{~m}$ ). A droite du pic, les tourbillons sont parsemés de bulles séparées ; à gauche, leur cœur est rempli de vapeur. En même temps, lorsque la cavitation se développe, l'écart relatif $b / a$ des deux allées passe de 0,23 à 0,07 environ.

\subsection{Détachement des poches de cavitation}

La question posée est relative à la position du détachement d'une cavité à partir d'une paroi à courbure continue, pour laquelle les critères de Villat-Armstrong (1911-1953) et Arakeri (1975), sont inopérants (voir [7] par exemple). Ce thème de travail a été étudié d'une manière quasi continue à l'IMG de 1980 à 1987 [6, 7, 9, 11]. Il a nécessité la prise en compte progressive des effets de couche limite et des effets de germes sur la cavitation par poches développées. Les méthodes utilisées dans cette recherche tendent à décrire le comportement global des écoulements : sur le plan théorique, les modèles utilisés sont ceux de poches [12, 13], de bulles [9], de couches limites [14] ; sur le plan expérimental, un grand nombre de renseignements ont été obtenus par des visualisations (injection de filets colorés) et par l'analyse de photographies ou de films rapides. Nous décrivons ici les principales étapes de la recherche et quelques-uns de ses principaux résultats.

Au cours de la première étape expérimentale (19801982), trois corps ont été étudiés : un cylindre circulaire (diamètre $D=1 \mathrm{~cm}$ ) et deux cylindres elliptiques (corde $c=8 \mathrm{~cm}$, rapports respectifs des demi-axes : $1 / 4$ et $1 / 8$ ). La position du détachement d'une cavité développée est a priori dépendante de deux paramètres principaux : le nombre de cavitation $\sigma_{v}$ et le nombre de Reynolds $\operatorname{Re}=V D / v$. Cette dernière dépendance est confirmée dans le cas du cylindre circulaire ; elle diminue nettement dans le cas du premier cylindre elliptique et disparaît pratiquement pour le corps le plus mince [6]. Le détachement de la cavité montre donc, dans le cas du corps mince, une parenté de comportement avec le décollement laminaire de la couche limite, dont on sait que la position est fixée uniquement par la répartition de pression et donc par la forme du corps.

Le lien entre le décollement laminaire de la couche limite et le détachement des poches de cavitation développée a été approfondi par FRANC de 1982 à 1985, au cours de campagnes d'essais d'un profil NACA 16012, de corde $10 \mathrm{~cm}$, maintenu d'abord fixe, de manière à réaliser un écoulement permanent, puis animé d'un mouvement d'oscillation en incidence (incidence moyenne $\bar{\alpha}=0,5$ et 10 degrés, amplitude 5 degrés, fréquence $f$ comprise entre 0 et $25 \mathrm{~Hz}$, fréquence réduite $f^{*}=f c / V$ pouvant atteindre l'unité). Un élément important pour la compréhension d'ensemble du phénomène a été l'établissement, par FRANC, de cartes des régimes cavitants dans le plan $\left(\alpha, \sigma_{v}\right)$, voir la figure 3 . Ces cartes dépendent peu du nombre de Reynolds, dans la limite des nombres de Reynolds étudiés $\operatorname{Re} \leqslant 1,1 \times 10^{6}$ ). On y remarque particulièrement la forme surprenante de la frontière entre les régimes cavitants et subcavitants et l'existence, aux petites incidences et aux faibles valeurs de $\sigma_{v}\left(\sigma_{v}<0,15\right)$, de cavités qui se détachent à l'arrière du corps. Dans ce cas, les particules de liquide traversent une zone où la pression est inférieure à $p_{v}$ et peut même être négative, ce qui n'est possible que si le liquide est exempt de germes de pression critique élevée. La forme en $S$ de la frontière implique qu'à une incidence voisine de 4 degrés la seule diminution de la pression ambiante entraîne d'abord l'apparition de la cavitation vers le bord d'attaque $\left(\sigma_{v} \simeq 0,7\right)$, son développement, puis sa disparition $\left(\sigma_{v} \simeq 0,35\right)$, enfin sa réapparition pour $\sigma_{v} \simeq 0,10$. Elle traduit la compétition entre le décollement laminaire de la couche limite, accompagné de la poche de cavitation, et la transition à la turbulence dans la couche limite. Le même phénomène peut être observé à des nombres de Reynolds plus grands, comme le montre la figure 4 , où sont portés des résultats d'essais de début de cavitation effectués récemment à l'Institut des machines hydrauliques et de mécanique des fluides de l'Ecole polytechnique fédérale de Lausanne. D'une manière générale, les observations de FRANC l'ont conduit à formuler le critère suivant :

— une cavité se détache à l'arrière d'un décollement laminaire ;

- la transition à la turbulence dans la couche limite supprime une cavité, même très développée.

Ce critère permet effectivement de prédire la position du détachement par des approximations successives qui combinent le calcul de l'écoulement potentiel et le calcul de la couche limite à l'amont de la poche. Dans le cas de l'écoulement non permanent autour de l'aile oscillante, le critère de détachement tient encore, pour toutes les réalisations expérimentales étudiées, mais son exploitation numérique ne paraît pas encore possible par défaut de modèle théorique de l'écoulement cavitant instationnaire $[11,15]$.

Dans la troisième étape de la recherche (1986-87) l'addition de germes à l'écoulement a permis d'étudier l'interaction entre couches limites, cavités et bulles [9], 


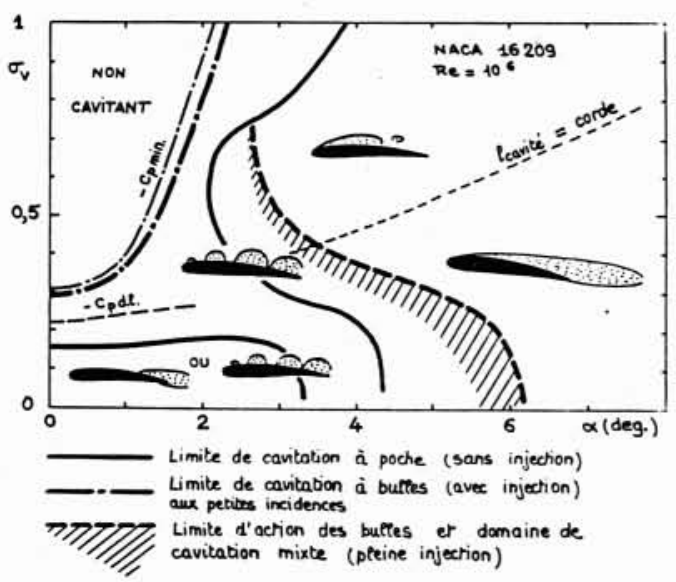

5.

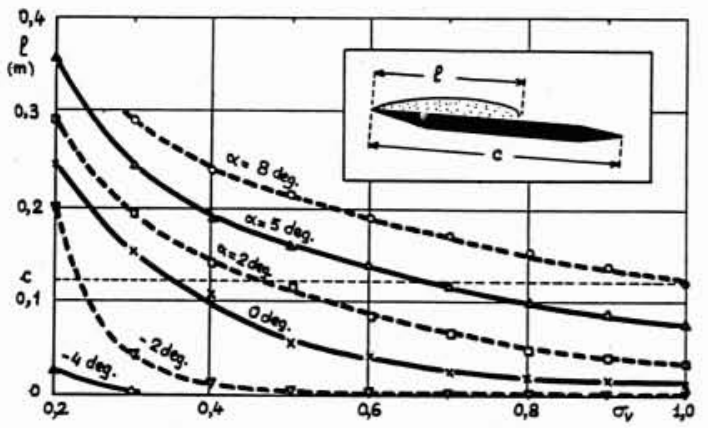

6.

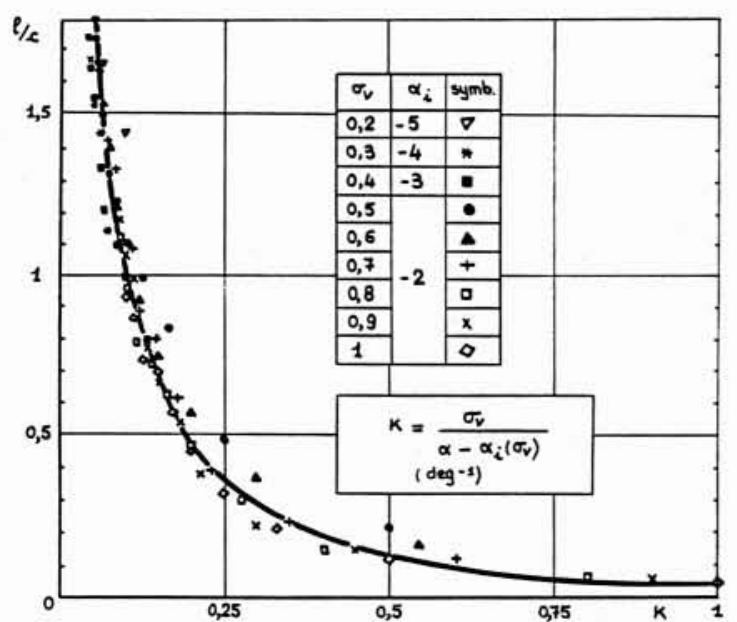

7.

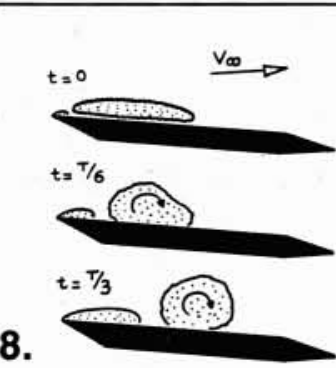

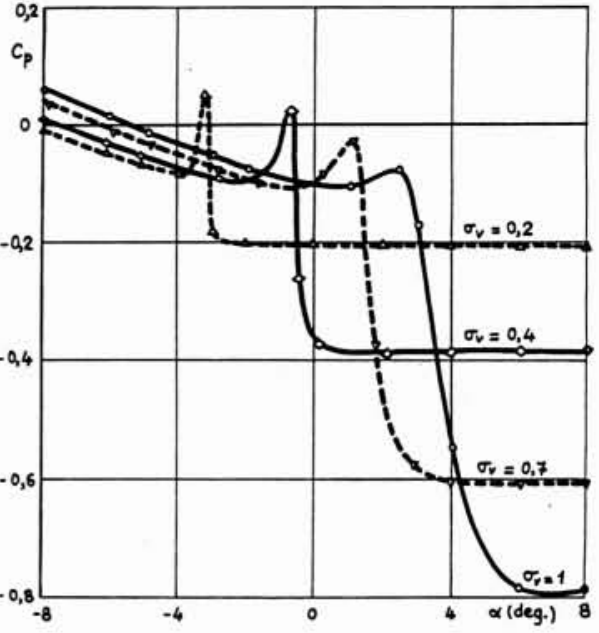

9.

5. Cavitation à bulles et cavitation par poches.

6. Longueur moyenne de la cavité d'extrados en fonction de l'incidence; profil $n^{\circ} 1$.

7. Regroupement des points de la figure 6 .

8. Evolution d'une cavité partielle périodique $\sigma_{v}=0,8$; $f=42,5 \mathrm{~Hz} ; \alpha=3 \mathrm{deg}$.

9. Coefficient de pression en un point fixe, d'abscisse $57 \mathrm{~mm}$, du profil $n^{\circ} 1 . V=10 \mathrm{~m} / \mathrm{s}$.

10. Répartitions de la pression à l'arrière de la poche sur le profil $n^{\circ} 2$.

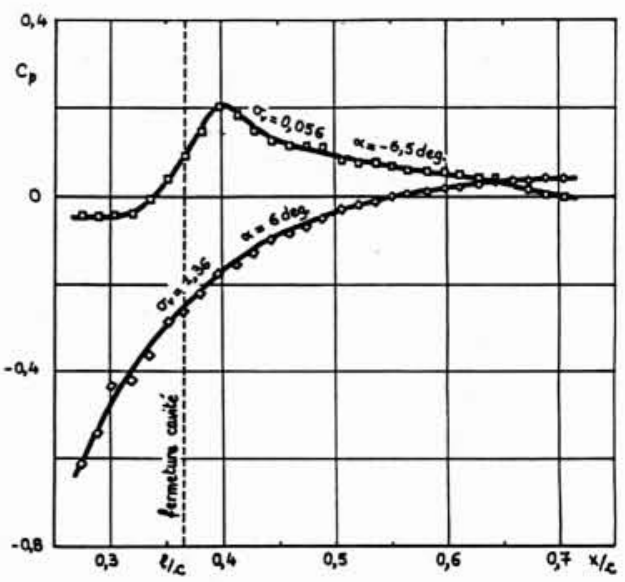

10. 
pour l'écoulement autour d'un profil NACA 16209. La figure 5 représente les domaines d'action respectifs des germes et des cavités. On note les points principaux suivants :

- les régimes de cavitation par poches, pour ce second profil, sont analogues à ceux du profil 16012. La forme en $S$ de la frontière entre écoulement cavitant et écoulement subcavitant est plus accentuée ;

- le régime de cavitation par bulles s'établit à des nombres $\sigma_{v}$ nettement plus grands, voisins de la valeur $-C_{p_{\min }}$, $\left(C_{p}\right.$ désigne le coefficient de pression), aux valeurs assez petites de l'incidence. Ces bulles naissent au voisinage du bord d'attaque et, lorsqu'elles sont assez nombreuses, elles empêchent la cavitation par poche d'apparaître à l'arrière du profil ;

- au contraire, pour les incidences plus élevées, les poches de cavitation se détachent elles-mêmes au bord d'attaque et empêchent les bulles d'exploser ;

- dans un domaine d'incidence moyenne, fonction décroissante de $\sigma_{v}$, les bulles et les cavités coexistent en une cavitation mixte. Le domaine s'élargit lorsque la concentration en germes injectés diminue.

L. BRIANÇON-MARJOLlET a étudié en détail les mécanismes par lesquels les bulles suppriment une cavité préexistante ou créent un état de saturation sur une partie du profil; elle a pu déduire de ses observations une estimation du nombre de germes nécessaires à l'un ou à l'autre phénomène [9].

Au total, l'ensemble des résultats expérimentaux obtenus laisse espérer que des règles de prédiction de la cavitation peuvent être établies qui tiennent compte des -principaux effets d'échelle, au moins dans le cas des écoulements assez voisins des configurations bi-dimensionnelles étudiées en laboratoire. Ces règles sont l'objet de la communication donnée à la présente session par $\mathrm{L}$. Briançon-Marjollet et J. P. Franc.

\subsection{Arrières de poches de cavitation partielle}

Les arrières de poches qui se referment sur une paroi solide sont intéressants à plusieurs titres : ils sont le siège de phénomènes complexes : jet rentrant, condensation, entraînement et implosion de poches de vapeur, d'où résultent des répartitions de pression moyenne dont les modélisations actuellement disponibles ne rendent compte qu'imparfaitement. Ils peuvent aussi donner lieu à une érosion intense. Il a alors paru utile d'étudier cette région d'écoulement sur des corps de forme simple, comportant en particulier un extrados plan. Cette forme permet l'implantation, à un coût peu élevé, de prises de pression moyenne et de capteurs de pics de pression qui sont décrits dans la suite. Notons que la mesure de la pression moyenne dans une région où sont présents successivement du liquide et de la vapeur ne va pas de soi. De ce fait, la campagne d'essais dont il est rendu compte, dont l'acteur principal a été M. NGUYEN THE Mich, étudiant vietnamien [16], avait pour but premier d'établir la faisabilité d'une telle mesure. Cela fait, plusieurs résultats intéressants ont été obtenus, qui sont approfondis actuellement par un second étudiant vietnamien, M. LE Quang. Des mesures analogues ont été faites récemment à Lausanne et leurs résultats vont sensiblement dans le même sens que ceux de l'I.M.G.

Un premier groupe de résultats est relatif à l'établissement des courbes donnant la longueur $\ell$ de la cavité en fonction de l'incidence $\alpha$ et du nombre de cavitation $\sigma_{v}$, pour le profil, dit profil $\mathrm{n}^{\circ} 1$, de corde voisine de $120 \mathrm{~mm}$, schématisé sur la figure 6 . Les résultats se regroupent assez bien lorsqu'on les présente en fonction de $\sigma_{v} /\left[\alpha-\alpha_{i}\left(\sigma_{v}\right)\right]$, où $\alpha_{i}\left(\sigma_{v}\right)$ désigne l'angle pour lequel la cavitation apparaît (fig. 7). Sur la figure 6, apparaît une zone où la longueur de la poche change beaucoup, à l'incidence constante, pour une très petite variation de $\sigma_{v}$. Cette tendance est retrouvée par les modèles théoriques de cavité partielle [13]. Elle traduit un comportement quasi instable de l'écoulement et est favorable à l'installation d'auto-oscillations.

La figure 8 représente la séquence des événements principaux qui se produisent lorsque l'auto-oscillation est périodique. Après qu'une cavité ait pris naissance au bord d'attaque, son développement se poursuit sur les $2 / 3$ de la période. Un jet rentrant, manifesté par la perturbation de la surface libre de la cavité, est créé à l'instant où la poche émise lors de la période précédente implose violemment. La vitesse du jet rentrant vers l'amont est à peu près égale à la vitesse de l'écoulement principal. La poche qui est issue de la cavité est alors le siège d'une circulation intense. Après que le jet rentrant ait atteint le bord d'attaque, la poche se gonfle beaucoup, puis implose. Sous l'influence des parois du canal, elle apparait alors assez souvent comme un filament tourbillonnaire dont l'axe est dans le plan médian du canal. Ces observations recoupent assez bien celles de KUBOTA et al. [17]. Selon ces derniers cependant, le caractère cyclique de l'écoulement est dû à l'instabilité de la frontière de la cavité et l'implosion subséquente des poches de vapeur ne semble y jouer aucun rôle.

La figure 9 montre l'évolution de la pression en un point fixe de l'aile, situé à $57 \mathrm{~mm}$ du bord d'attaque, en fonction de l'incidence. Un maximum y apparaît lorsque la fermeture de la poche est pratiquement au droit de la prise de pression. La figure 10 montre la répartition de pression correspondante obtenue récemment par LE Quang sur l'extrados plan d'un profil, de corde voisine de $200 \mathrm{~mm}$, dont l'indrados est circulaire. La remontée de la pression à l'arrière de la poche est très nette ; un maximum dans la répartition spatiale de $C_{p}$ existe effectivement pour les plus petites valeurs de $\sigma_{v}$. Les deux répartitions de pression sont obtenues pour la même valeur de la longueur de la cavité, telle que $l / c \simeq 0,37$.

Plusieurs mesures de pics de pression à l'arrière des poches ont été effectuées par NGUYEN THE et les histogrammes correspondants ont été réalisés. Le nombre total de pics en un point donné de l'aile passe par un maximum lorsque l'arrière de la poche est un peu à l'amont du point d'observation. Dans les cas expérimentaux étudiés $\left(V=10 \mathrm{~m} / \mathrm{s}, \sigma_{v}=0,5,0,7\right.$ et 0,9$)$ la distance est de l'ordre de $1 \mathrm{~cm}$ et le nombre maximal de pics est d'environ 30 par $\mathrm{mm}^{2}$ et par seconde. 


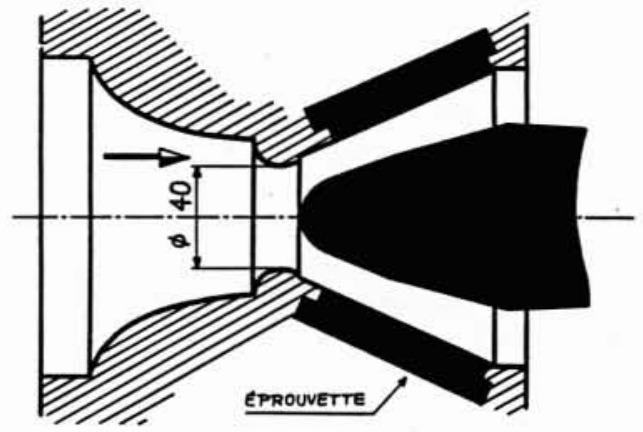

11.

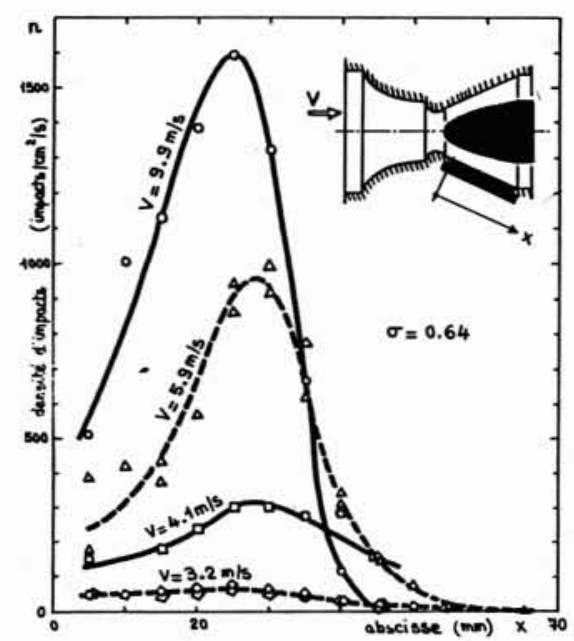

12.

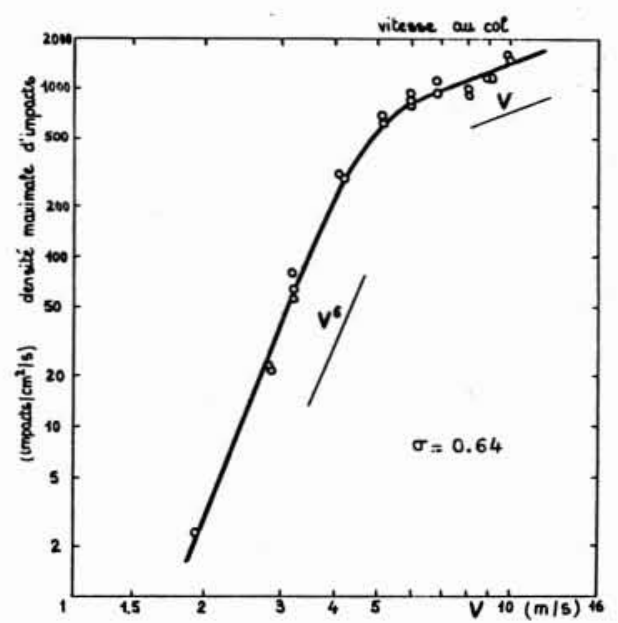

13.

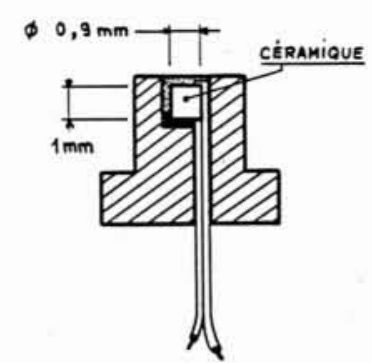

14.

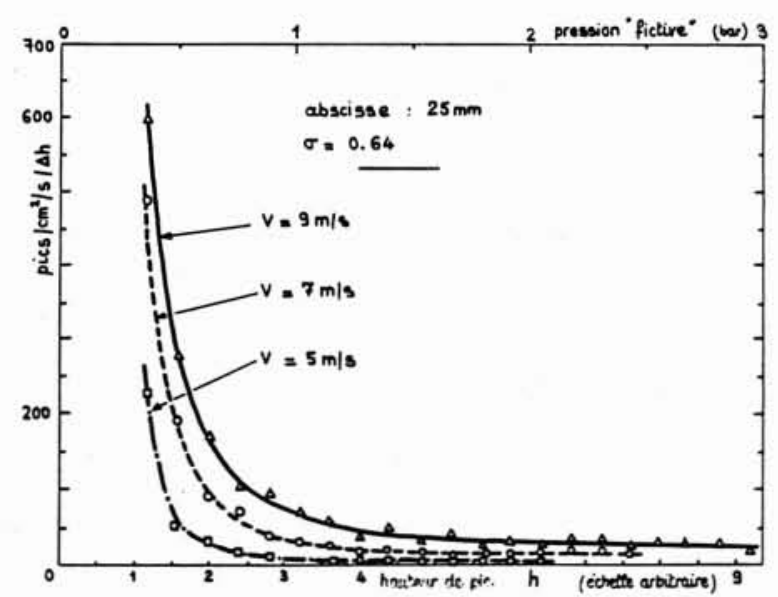

15.

11. Section d'essai de la boucle à mercure.

12. Essais au mercure. Distribution de la densité d'impacts le long de l'abscisse de l'éprouvette. Influence de la vitesse.

13. Essais au mercure. Evolution de la densité maximale d'impacts en fonction de la vitesse $(x=25 \mathrm{~mm})$.

14. Schéma d'un capteur.

15. Essais en eau. Histogramme de pics de pression à l'abscisse $x=25 \mathrm{~mm}$.

16. Essais en eau. Histogrammes réduits des pics de pression.

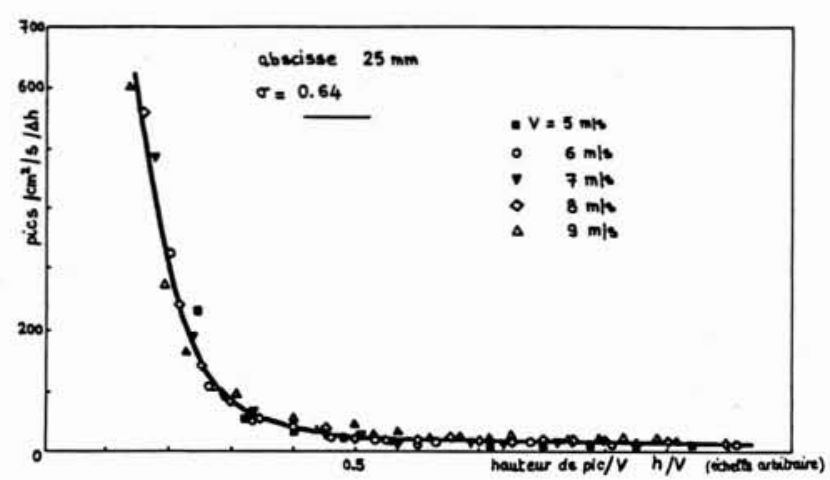

16. 


\section{Erosion de cavitation}

Le thème de l'érosion de cavitation a été abordé depuis 1982 à l'IMG, par une opération menée de concert avec le CERG, et financée par la DRET, à laquelle EDF s'est ensuite associée. Il s'agissait au départ de tester les hypothèses de similitude émises par Y. LECOFFRE sur la capacité érosive des écoulements cavitants [19]. A cette fin, une boucle à mercure a été construite et mise au point en 1983-84. Les essais d'érosion y ont été effectués en 1984-85 par FRANC et ils seront prochainement repris et complétés. Dans ces essais, l'éprouvette-témoin joue le rôle d'un capteur à seuil. Il a paru alors intéressant de mener sur le même site, la boucle fonctionnant alors en eau, des comptages de pics de pression au moyen de petits capteurs construits au laboratoire. Nous présentons ci-dessous la boucle d'essais et les divers résultats obtenus.

\subsection{La boucle d'essais au mercure}

La boucle d'essais au mercure comprend les principaux éléments suivants :

- une pompe volumétrique dont le débit maximum est $13 \mathrm{l} / \mathrm{s}$

- un accumulateur qui amortit les fluctuations de débit

— un échangeur de chaleur

— un bidon pressurisé à l'azote pour contrôler la pression.

Le volume total de mercure est de 86 litres soit une masse voisine de $1170 \mathrm{~kg}$.

La section d'essais (fig. 11) est constituée d'un venturi de diamètre $40 \mathrm{~mm}$. Elle a été conçue par le CERG de manière à produire une cavité attachée à la paroi à l'aval d'un décollement laminaire et à obtenir un écoulement stable avec un fort gradient adverse de la pression. La vitesse moyenne maximale au col est $10 \mathrm{~m} / \mathrm{s}$. Selon les hypothèses de LECOFFRE, les structures de vapeur ont alors une intensité du même ordre que celles d'un écoulement d'eau géométriquement semblable de vitesse voisine de $130 \mathrm{~m} / \mathrm{s}$.

\subsection{Résultats d'essais d'érosion au mercure}

La figure 12 montre, à plusieurs vitesses, la distribution de densité d'impacts le long de l'abscisse de l'éprouvette. Le maximum se produit à une abscisse sensiblement constante, le nombre de cavitation étant gardé constant. Le résultat majeur est porté sur la figure 13 qui montre l'évolution de maximum de la densité d'impacts en fonction de la vitesse [13]. Pour les vitesses les plus élevées $(V>5,5 \mathrm{~m} / \mathrm{s}$, correspondant à des vitesses en eau supérieures à $70 \mathrm{~m} / \mathrm{s}$ environ) la croissance est du type $V^{n}$ avec $n \simeq 1$, ce qui correspond aux prédictions du modèle de Lecoffre. On note aussi que des impacts sont produits à la vitesse minimale alors permise par l'installation, soit $2 \mathrm{~m} / \mathrm{s}$.

\subsection{Capteurs de pics de pression}

La mesure des pics de pression à l'arrière d'une poche de vapeur se heurte à plusieurs difficultés [20] :
- leur étendue réelle $\Delta S$ est petite par rapport à la taille $\Delta S^{\prime}$ du capteur et demeure de toute manière inconnue. Le signal de sortie du capteur, ne peut alors être traduit rigoureusement qu'en terme de force $F$, d'où résulte une pression fictive $p^{\prime}=F / \Delta S^{\prime}$, la pression réelle $p$ étant reliée à $p^{\prime} \operatorname{par} p / p^{\prime} \simeq \Delta S^{\prime} / \Delta S$;

- leur temps caractéristique est très petit, sans doute inférieur à la microseconde. Le capteur et la chaîne de mesure doivent alors une fréquence propre supérieure au mégahertz sinon l'amplitude de sortie est réduite dans un rapport à peu près égal à celui des fréquences ;

- leur amplitude est très élevée, lorsque la vitesse de l'écoulement est grande, ce qui risque de détruire le capteur.

Le parti pris à l'IMG depuis 1983 a été de fabriquer les capteurs au laboratoire à partir de céramiques piézoélectriques (diamètre $0,9 \mathrm{~mm}$, épaisseur $1 \mathrm{~mm}$, fréquence propre $1,8 \mathrm{MHz}$ ) travaillant en compression et logées dans un boîtier en plexiglas, voir figure 14. Dans un premier temps [16], l'amplification du signal était réalisée par une chaîne ancienne existant au laboratoire dont la fréquence propre était au plus de $280 \mathrm{KHz}$. Plus récemment, on a utilisé des amplis de charge de fréquence propre $2 \mathrm{MHz}$. Le montage des céramiques nécessite un étalonnage dynamique préalable. Nous effectuons un étalonnage relatif à faible fréquence et petite amplitude d'excitation. De plus, des essais de chocs par chute de billes, bien que d'interprétation délicate, ont montré que les valeurs des sensibilités obtenues sont encore significatives pour les grandes amplitudes et les grandes fréquences.

\subsection{Résultats de mesures de pics de pression}

Les hypothèses de LECOFFRE impliquent que, à nombre de cavitation égal et à vitesse égale, le nombre de structures de vapeur émises par une poche de cavitation partielle soit le même en mercure et en eau. Si l'on suppose de plus que la dynamique de ces structures est sensiblement la même entre la zone d'émission et la zone d'implosion sur la paroi, on est fondé à penser que le nombre total de pics de pression significatifs en écoulement d'eau doit être du même ordre que le nombre total d'impacts permanents exercés par le mercure sur les éprouvettes, au moins dans la gamme des vitesses grandes pour lesquelles toutes les structures de vapeur émises par la cavité sont efficaces. Les essais effectués en eau avec un capteur de pression placé à l'abscisse d'érosion maximale tendent alors à vérifier cette hypothèse. Dans la pratique, un problème supplémentaire se pose, celui du seuil de détection des pics du signal de sortie de vapeur. Le seuil a d'abord été fixé de manière à ce que, à la plus grande vitesse, soit $10 \mathrm{~m} / \mathrm{s}$, le nombre de pics soit égal au nombre d'impacts, soit environ $1500 \mathrm{~cm}^{2} / \mathrm{s}$. Plus récemment l'effet de la valeur du seuil a été systématiquement étudié.

Dans la première étape de la recherche [16], il a été vérifié que le nombre total de pics suit approximativement la même évolution que le nombre total d'impacts pour les vitesses comprises entre 5 et $10 \mathrm{~m} / \mathrm{s}$. Des histogrammes de pics de "pression fictive $p^{\prime}$ » ont été établis (fig. 15). La figure 16 montre que les résultats se rassemblent à peu près en une seule courbe lorsqu'on 


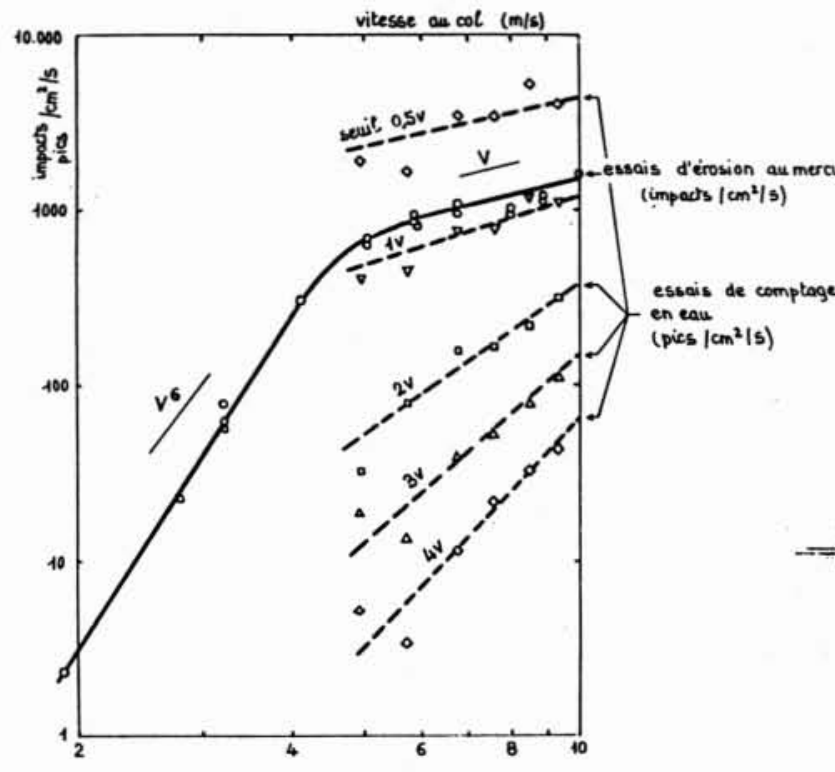

18.

réduit les abscisses par la vitesse $V$ : ce résultat est lui aussi conforme aux hypothèses de LECOFFRE.

La dernière campagne d'essais, menée au cours de l'automne 87, a utilisé un capteur suivi d'un ampli de charge haute fréquence. On peut donc espérer que l'atténuation d'amplitude due à l'instrumentation n'est pas grande. La figure 17 montre l'allure du signal. Les histogrammes obtenus ont la même allure que ceux de la figure 15. Finalement, l'effet du seuil est étudié sur la figure 18. Le seuil de 1 volt donne à peu près les mêmes résultats que le nombre total d'impacts dus au mercure, dans la gamme de vitesse $5-10 \mathrm{~m} / \mathrm{s}$. Un seuil inférieur fait apparaître une plus grande quantité de pics, dont il est difficile de savoir si certains sont dus à des échos ou à des structures de vapeur implosant dans le voisinage du capteur. Enfin, la pente des courbes correspondant aux seuils plus élevés est plus grande que l'unité. Ces courbes, qui simulent l'action du mercure sur un métal plus résistant que l'acier Inox $316 \mathrm{~L}$ pris comme référence, ont un comportement qui satisfait aussi aux hypothèses de LECOFFRE. Cependant, l'étroitesse du domaine de vitesses considéré ici empêche de tirer des conclusions définitives.

\section{Conclusion}

Les recherches décrites dans l'article sont poursuivies actuellement à l'IMG, dans les deux domaines de la cavitation partielle et de l'agressivité des écoulements cavitants. Par ailleurs, un rapport d'ensemble concernant l'érosion de cavitation a été rédigé [21] suite aux réunions du groupe de travail "Cavitation » de la SHF et à la demande de la DRET, d'EDF et du CNRS. Ce
17. Essais en eau. Allure du signal $V=9,1 \mathrm{~m} / \mathrm{s}, \sigma_{v}=0,63$. Taux d'acquisition : $20 \mathrm{MHz}$.

18. Evolution du nombre total des pics en fonction de la vitesse. Effet du seuil de détection.

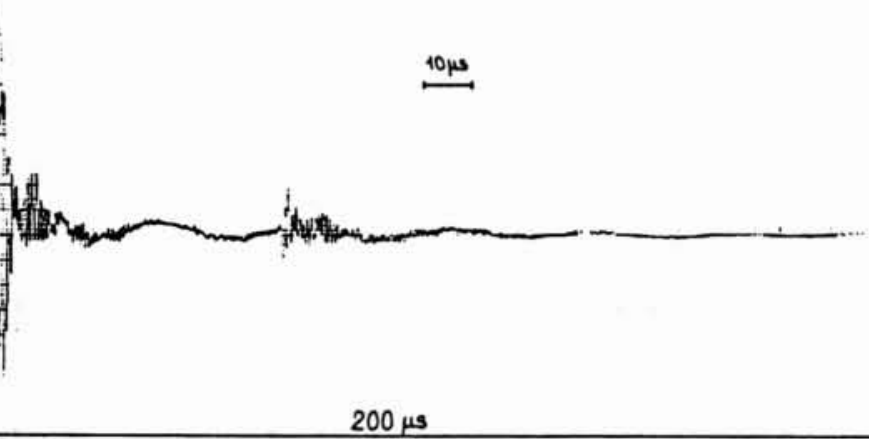

rapport, fruit des réflexions menées par plusieurs personnes appartenant à des laboratoires de recherche ou à des organismes industriels divers, avait pour objet de tracer un tableau des connaissances acquises, de situer les recherches entreprises en France sous le patronage de la DRET, d'identifier les principaux points durs du problème, de proposer quelques actions pour le court terme et d'imaginer, autant qu'il est possible, des axes de recherche pour les années à venir. Plusieurs actions proposées sont déjà en cours de réalisation. En particulier, les idées de LECOFFRE sur la similitude hydrodynamique de l'érosion de cavitation, que nous savons assez largement confirmées par les travaux déjà effectués en laboratoire, pourront trouver un lieu d'application à la prédiction industrielle de l'érosion lorsque le «CAVERSIM " industriel (voir [19]), aura été réalisé. La création d'un appareil de laboratoire destiné à produire des impacts érosifs contrôlés est aussi envisagée ; on peut espérer qu'un tel appareil sera un outil d'analyse efficace pour les mécaniciens du solide et les métallurgistes.
Adresse de l'auteur:

Monsieur J. M. Michel

IMG

Domaine universitaire

BP $53 \mathrm{X}$

38041 Grenoble Cedex

Tél. : (16) 76825000 


\section{Références}

[1] Dodu J., Duport J., Michel J. M. : « Le Tunnel Hydrodynamique de l'Université de Grenoble ", La Houille Blanche, $1968, \mathrm{n}^{\circ} 7$, pp. 697-702.

[2] Dodu J. : *Contribution à l'étude de la dispersion des jets liquides à grande vitesse ", Publications scientifiques et techniques du Ministère de l'Air, n 407, 1964.

[3] Danel. P., Duport J. : « The selection of length and head scales for cavitation tests ", J. of Basic Eng., 82, Ser. D, 1960 , pp. $784-790$.

[4] Michel. J. M., Rowe A. : * Profils minces supercavitants à arrière tronqué ", La Houille Blanche, 1974, n³, pp. 205 230 et $n^{*} 6$, pp. $429-445$.

[5] Dodu J., Michel J. M., Rowe A. : « Les écoulements autour des ailes ventilées ", La Houille Blanche, 1977. n" 5-6, pp. 449-458.

[6] Michel J. M., Biset S., Franc J. P. : « Cavitation développée sur des parois à courbure continue: Physique du détachement ", La Houille Blanche, n* 7-8, 1982, pp. 559 570 .

[7] FRANC J. P., MiChEL J. M. : « Attached cavitation and the boundary layer : experimental investigation and numerical treatment ", J. of Fluid Mechanics (1985), vol. 154, pp. 63 90.

[8] Briançon-Marjollet L., Michel J. M. : « The hydrodynamic tunnel of IMG : former and recent equipments ", Int. Symp. on Cavitation Research Facilities and Techniques. Boston, 13-18 déc. 1987, Proc., pp. 37-47.

[9] BrianÇON-MARJollet L. : "Couches limites, germes et cavités en interaction: Etude physique ». Thèse, Grenoble, octobre 1987.

[10] Franc J. P. : * Etudes de cavitation. II. Sillages d'obstacles épais. Etude physique ». Thèse de D.I., Grenoble, septembre 1982.

[11] Franc J. P. : « Etude physique d'écoulements cavitants ». Thèse d'Etat, Grenoble, mars 1986.
[12] Pellone C., Rowe A. : « Supercavitating hydrofoils in nonlinear theory ", 3rd Int. Conf. on Num. Ship Hydrodynamics. Paris, 1981, Proc., pp. 399-412.

[13] Lemonnier H.: *Optimisation du maillage et de la répartition de simple couche dans la méthode des singularités ». Thèse de D.I., Grenoble, janvier 1984.

[14] Arnal D., Habiballah M., Coustols E. : « Théorie de l'instabilité laminaire et critère de transition en écoulement bi et tri-dimensionnel ", La Recherche Aérospatiale, vol. 1984-2.

[15] Franc J. P., MiChel J. M. : « Unsteady attached cavitation on an hydrofoil oscillating foil n, J. of Fluid Mechanics (1988), vol. 193, pp. 171-189.

[16] NGUYEN THE Mich. : « Fermeture de poches de cavitation partielle: Cinématique. Pressions à la paroi $»$. Thèse de D.I., Grenoble, octobre 1986.

[17] Kubota S., Kato H., Yamaguchi H., Maeda M. * Unsteady structure measurements of cloud cavitation on a foil section using conditional sampling techniques ", Int. Symp. on Cavitation Research Facilities and Techniques. Boston, 13-18 déc. 1987, Proc., pp. 161-168.

[18] Nguyen The M., Franc J. P., Michel J. M. : «On correlating pitting rate and pressure peak measurements in cavitating flows ", Int. Symp. on cavitation Res. Facilities and Techniques. Boston, 13-18 déc. 1987, Proc., pp. $207-$ 216.

[19] Amblard A., Bonazzi A., Lecoffre Y. : "Développements récents d'une méthode de prévision de l'érosion de cavitation" SHF, Colloque d'Hydrotechnique "Cavitation ». Paris, 22-23 mars 1988, n 12.

[20] Paulon J. : « Evolution récente de l'appareillage et des techniques de mesures en aérodynamique et en hydrodynamique ", La Houille Blanche, 1987, n 6, pp. 411-445.

[21] MiChel J. M., CHIEM C. Y. : * Lignes de recherches en érosion de cavitation ", Rapport action concertée CNRSDRET-EDF, 87 p., Grenoble, juillet 1986. 
M. AVELLAN : Peut-on mettre de l'ordre dans la cavitation développée à grand nombre de Reynolds ?

Il me semble qu'il y a 2 régimes de dynamique de fermeture des poches :

1 régime à poches fines,

1 régime à grosses poches à jet rentrant et présentant d'importantes fluctuations d'ensembles.

Un point essentiel, si l'on veut aller plus loin serait de préciser la nature des instabilités.

M. MICHEL : La longueur de cavité, relativement au profil, n'est peut-être pas seule à intervenir. Son épaisseur relative est sans doute aussi importante. Mais il est difficile de quantifier ce paramètre d'une manière précise.

M. AVELLAN : Dans les expériences que nous avons faites, nous sommes toujours restés très en deçà du point d'arrêt.

Il semble, dans votre cas que vous montiez assez fortement en pression. Vous rapprochez-vous de la pression point d'arrêt ?

M. MICHEL : Dans notre cas, les poches sont très aplaties, avec peu d'oscillations.

M. AVELLAN: Avez-vous étalonné vos capteurs de pression pour caler les seuils des impacts?

M. MICHEL: Notre seuil correspond approximativement à 0,3 bar en pression fictive. La grande inconnue reste la surface de l'impact.

Mme NIENALTOWSKA : Une recherche concernant la corrélation entre les vitesses d'érosion et le nombre de pics (signaux acoustiques) supérieurs à un niveau seuil a été effectuée à Southampton, par les collaborateurs du professeur HUTTON. Un des problèmes a été le choix de ce seuil, mais aussi l'emplacement des capteurs par rapport à la fin de la poche de façon à obtenir un nombre maximum de pics.

Avez-vous déterminé la position de la zone de fermeture de poche par rapport à la zone où la densité de pics est la plus importante?
M. MICHEL: Une réponse partielle nous a été fournie par les essais de M. N'GUYEN TE MiCH, qui a trouvé un maximum un peu à l'arrière de la fermeture de poche, à environ $1 \mathrm{~cm}$.

Ces mesures sont actuellement reprises par M. LE QUANG, avec un ensemble de 9 capteurs.

Mme NIENALTOWSKA : Je reviens au problème du seuil. Dans le cas que j'ai cité, le seuil pour le comptage de pics a été ajusté par rapport aux résultats des essais de l'érosion de façon à obtenir une loi analogue en fonction de la vitesse de l'écoulement. Ainsi, on a pu retrouver, a posteriori, des bonnes corrélations entre les deux phénomènes. Cependant, pour une géométrie différente il faut toujours se référer aux résultats de l'érosion.

M. DESCLAUX : Quelle explication donnez-vous aux deux pentes de la courbe donnant le nombre d'impacts en fonction de la vitesse?

M. MICHEL : L'explication la plus simple est celle donnée par M. LeCOFFre. Elle suppose que l'histogramme d'intensité des impacts, qui se déplace sous l'effet de la vitesse ait un seuil haut et un seuil bas. Lorsque la vitesse est suffisante pour que les cavités les moins agressives marquent le matériau, l'évolution du nombre d'impacts devient proportionnelle à la vitesse. Les lois de similitude sont très simples.

M. DESCLAUX : Vous avez employé le mot de "saturation ".

M. MICHEL: Il s'agissait alors de cavitation développée par bulles et non d'érosion.

M. DESCLAUX: Une hypothèse qui peut être émise est, du fait de la présence de vapeur dans le liquide, donc de diphasique, une réduction de la vitesse du son dans l'écoulement. Celle-ci peut générer, si elle est suffisante, un phénomène de blocage sonique. Un moyen de vérifier est de comparer des courbes à différents nombres de cavitation.

M. MICHEL: Il faudrait faire beaucoup d'essais. Peut-être ya-t'il des moyens plus simples, la mesure des volumes de poche par exemple?

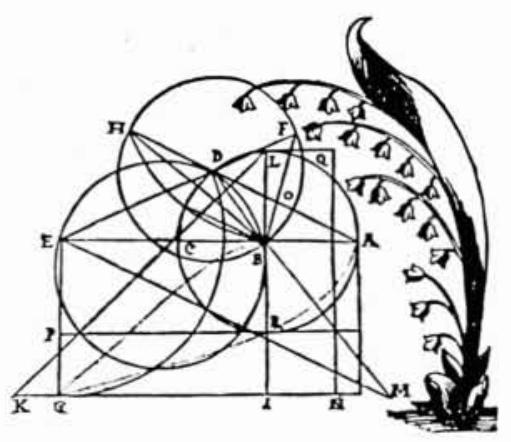

